\title{
Field Emission from Laser Cut CNT Fibers and Films- CORRIGENDUM
}

\author{
Steven B. Fairchild, John S. Bulmer, Martin Sparkes, John Boeckl, Marc Cahay, Tyson Back, \\ P. Terrence Murray, Gregg Gruen, Matthew Lange, Nathaniel P. Lockwood, Francisco Orozco, \\ William O’Neill, Catharina Paukner, and Krzysztof K. K. Koziol
}

doi: 10.1557/jmr.2013.322, Published by Materials Research Society with Cambridge University Press, 11 November 2013.

In Fairchild et al., ${ }^{1}$ Gregory Kozlowski was omitted from the author list. The corrected list of authors and affiliations appears below.

Steven B. Fairchild

Materials and Manufacturing Directorate, Air Force Research Laboratory, Wright-Patterson Air Force Base,

Ohio 45433

John S. Bulmer

Department of Materials Science and Metallurgy, University of Cambridge, Cambridge CB3 OFS, UK

Martin Sparkes

Department of Engineering, Institute for Manufacturing, University of Cambridge, Cambridge CB3 OFS, UK

John Boeckl

Materials and Manufacturing Directorate, Air Force Research Laboratory, Wright-Patterson Air Force Base,

Ohio 45433

Marc Cahay

Spintronics and Vacuum Nanoelectronics Laboratory, University of Cincinnati, Cincinnati, Ohio 45221

Tyson Back and P. Terrence Murray

Research Institute, University of Dayton, Dayton, Ohio 45469-0170

Gregg Gruen and Matthew Lange

TechFlow Scientific, Albuquerque, New Mexico 87110

Nathaniel P. Lockwood

Directed Energy Directorate, Air Force Research Laboratory, Kirtland Air Force Base, New Mexico 87117

Francisco Orozco

Department of Engineering, Institute for Manufacturing, University of Cambridge, Cambridge CB3 OFS, UK

William O'Neill

Department of Engineering, Institute for Manufacturing, University of Cambridge, Cambridge CB3 OFS, UK

Catharina Paukner and Krzysztof K. K. Koziol

Department of Materials Science \& Metallurgy, University of Cambridge, Cambridge CB3 OFS, UK

Gregory Kozlowski

Department of Physics, Wright State University, Dayton, Ohio 45435

The authors regret the mistake.

\section{REFERENCE}

1. S.B. Fairchild, J.S. Bulmer, M. Sparkes, J. Boeckl, M. Cahay, T. Back, P.T. Murray, G. Gruen, M. Lange, N.P. Lockwood, F. Orozco, W. O’Neill, C. Paukner, and K.K.K. Koziol: Field Emission from Laser Cut CNT Fibers and Films. J. Mater. Res. (2013).

DOI: $10.1557 /$ jmr.2014.10 